\title{
DEFORMATIONS OF CONVOLUTION SEMIGROUPS ON COMMUTATIVE HYPERGROUPS
}

\author{
MARGIT RÖSLER \\ Mathematisches Institut, Universität Göttingen \\ Bunsenstr. 3-5, D-37073 Göttingen, Germany \\ E-mail: roesler@uni-math.gwdg.de \\ MICHAEL VOIT \\ Fachbereich Mathematik, Universität Dortmund \\ D-44221 Dortmund, Germany \\ E-mail: michael.voit@mathematik.uni-dortmund.de
}

\begin{abstract}
It was recently shown by the authors that deformations of hypergroup convolutions w.r.t. positive semicharacters can be used to explain probabilistic connections between the Gelfand pairs $(S L(d, \mathbb{C}), S U(d))$ and Hermitian matrices. We here study connections between general convolution semigroups on commutative hypergroups and their deformations. We are able to develop a satisfying theory, if the underlying positive semicharacter has some growth property. We present several examples which indicate that this growth condition holds in many interesting cases.
\end{abstract}

\section{Introduction}

Klyachko ${ }^{8}$ recently derived a connection between $S U(d)$-biinvariant random walks on $S L(d, \mathbb{C})$ and random walks on the additive group $\mathbf{H}_{d, 0}$ of all hermitian $d \times d$-matrices with trace 0 , whose transition probabilities are invariant under conjugation by $S U(d)$. He used this connection to transfer the recent solution of the spectral problem for sums of hermitian matrices $\left({ }^{7},{ }^{10}\right)$ to the possible singular spectrum of products of random matrices from $S L(d, \mathbb{C})$ with given singular spectra. The singular spectrum of a matrix $A \in S L(d, \mathbb{C})$ here means the spectrum of the positive definite matrix $\sqrt{A A^{*}}$. Klyachko's connection between $S L(d, \mathbb{C})$ and $\mathbf{H}_{d, 0}$ was explained in a different way and extended by the authors in ${ }^{18}$; it is shown in 18 that the commutative Banach algebra of all $S U(d)$-biinvariant bounded measures on $S L(d, \mathbb{C})$ may be embedded into the Banach algebra of all bounded measures on the Euclidean space $\mathbf{H}_{d, 0}$ in an isometric, probabil- 
ity preserving way. The proof of this fact, which has some applications in probability theory (see ${ }^{18}$ ), depends on so-called deformations of hypergroup convolutions with respect to positive semicharacters as introduced in ${ }^{20}$. These deformations lead to connections between random walks and convolution semigroups on different, but closely related hypergroups. This forms the motivation to investigate systematically when and how convolution semigroups of probability measures on a commutative hypergroup $(X, *)$ can be transformed canonically into convolution semigroups on a deformation $(X, \bullet)$ of $(X, *)$. In particular we show that the generators and Lévy measures of the original and the deformed convolution semigroup are closely related whenever this transformation is possible. We mention that the deformation of convolution semigroups is closely related to Doob's $h$-transform, and that Lévy processes associated with a convolution semigroup and its deformation are related by a Girsanov transformation on the path space; see ${ }^{21}$.

The paper is organized as follows: In Section 2 we collect some facts on deformations and present examples. In particular we indicate how for a maximal compact subgroup $H$ of a complex, non-compact, connected semisimple Lie group $G$, the double coset hypergroup $G / / H$ may be regarded as deformation of an orbit hypergroup. This includes the examples above. Section 3 is devoted to deformations of convolution semigroups w.r.t. positive semicharacters $\alpha_{0}$. We show that this concept works in a satisfying way under a canonical growth condition on the convolution semigroup together with some growth condition concerning $\alpha_{0}$. Section 4 finally contains examples where this condition on $\alpha_{0}$ is satisfied. In fact, we have no example for which this condition would not hold.

\section{Deformations of commutative hypergroups}

We give a quick introduction. First, let us fix notations. For a locally compact Hausdorff space $X, M^{+}(X)$ denotes the space of all positive Radon measures on $X$, and $M_{b}(X)$ the Banach space of all bounded regular complex Borel measures with the total variation norm. Moreover, $M^{1}(X) \subset M_{b}(X)$ is the set of all probability measures, $M_{c}(X) \subset M_{b}(X)$ the set of all measures with compact support, and $\delta_{x}$ the point measure in $x \in X$. The spaces $C(X) \supset C_{b}(X) \supset C_{0}(X) \supset C_{c}(X)$ of continuous functions are given as usual.

Definition 2.1. A hypergroup $(X, *)$ consists of a locally compact Hausdorff space $X$ and a weakly continuous, probability preserving convolution 
* on $M_{b}(X)$ such that $\left(M_{b}(X), *\right)$ is a Banach algebra and $*$ preserves compact supports. Moreover, there exists an identity $e \in X$ (such that $\delta_{e}$ is the identity of $\left.\left(M_{b}(X), *\right)\right)$ as well as a continuous involution $x \mapsto \bar{x}$ on $X$ that replaces the group inverse. For details we refer to ${ }^{1}$ and ${ }^{6}$.

We here only deal with commutative hypergroups $(X, *)$, i.e., $*$ is commutative. In this case there exists an (up to normalization) unique Haar measure $\omega \in M^{+}(X)$ which is characterized by $\omega(f)=\omega\left(f_{x}\right)$ for all $f \in C_{c}(X)$ and $x \in X$, where we use the notation

$$
f_{x}(y):=f(x * y):=\int_{X} f d\left(\delta_{x} * \delta_{y}\right) .
$$

Similar to the dual of a locally compact abelian group, one defines

$$
\begin{aligned}
\chi(X) & :=\{\alpha \in C(X): \alpha \neq 0, \alpha(x * y)=\alpha(x) \alpha(y) \text { for all } x, y \in X\}, \\
X^{*} & :=\{\alpha \in \chi(X): \alpha(\bar{x})=\overline{\alpha(x)} \text { for } x \in X\} ; \quad \widehat{X}:=X^{*} \cap C_{b}(X) .
\end{aligned}
$$

Elements of $X^{*}$ and $\widehat{X}$ are called semicharacters and characters respectively. All spaces are locally compact w.r.t. the compact-uniform topology.

\section{Example 2.1.}

(1) Let $K$ be a compact subgroup of a locally compact group $G$. Then

$$
M_{b}(G \| K):=\left\{\mu \in M_{b}(G): \delta_{x} * \mu * \delta_{y}=\mu \text { for all } x, y \in K\right\}
$$

is a Banach-*-subalgebra of $M_{b}(G)$ with the normalized Haar measure $d k \in M^{1}(G)$ of $K$ as identity. The double coset space $G / / K:=$ $\{K x K: x \in G\}$ is locally compact w.r.t. the quotient topology, and the canonical projection $p: G \rightarrow G / / K$ induces a probability preserving, isometric isomorphism $p: M_{b}(G \| K) \rightarrow M_{b}(G / / K)$ of Banach spaces by taking images of measures. The transport of the convolution on $M_{b}(G \| K)$ to $M_{b}(G / / K)$ via $p$ leads to a hypergroup structure $(G / / K, *)$ with identity $K$ and involution $(K x K)^{-}:=K x^{-1} K$, and $p$ even becomes a Banach- $*$-algebra isomorphism. If $G / / K$ is commutative, i.e., $(G, K)$ is a Gelfand pair, then a $K$-biinvariant function $\varphi \in C(G)$ with $\varphi(e)=1$ is spherical if $\varphi(x) \varphi(y)=\int_{K} f(x k y) d k$ for $x, y \in G$. The functions $\alpha \in \chi(G / / K)$ are in one-to-one correspondence with the spherical functions on $G$ via $\alpha \mapsto \alpha \circ p$ for the canonical projection $p: G \rightarrow G / / K$.

(2) Let $(V,\langle.,\rangle$.$) be a finite-dimensional Euclidean vector space and$ $K \subset O(V)$ a compact subgroup of the orthogonal group of $V$. For 
$\mu \in M_{b}(V)$, denote the image measure of $\mu$ under $k \in K$ by $k(\mu)$. Then the space of $K$-invariant measures

$$
M_{b}^{K}(V):=\left\{\mu \in M_{b}(V): k(\mu)=\mu \text { for all } k \in K\right\}
$$

is a Banach-*-subalgebra of $M_{b}(V)$ (with the group convolution) with identity $\delta_{0}$. The space $V^{K}:=\{K . x: x \in V\}$ of all $K$-orbits in $V$ is again locally compact, and the canonical projection $p$ : $V \rightarrow V^{K}$ induces a probability preserving, isometric isomorphism $p: M_{b}^{K}(V) \rightarrow M_{b}\left(V^{K}\right)$ of Banach spaces and an associated orbit hypergroup structure $\left(V^{K}, *\right)$ such that $p$ becomes an isomorphism of Banach-*-algebras. The involution on $V^{K}$ is given by $\overline{K . x}=$ $-K . x$. Moreover, the continuous functions

$$
\alpha_{\lambda}(K . x)=\int_{K} e^{i\langle\lambda, k . x\rangle} d k \quad(x \in V)
$$

are multiplicative on $\left(V^{K}, *\right)$ for $\lambda \in V_{\mathbb{C}}$, the complexification of $V$, and $\alpha_{\lambda} \equiv \alpha_{\mu}$ if and only if $K . \lambda=K$. $\mu$. It is known $\left(\right.$ see $^{6}$ ) that $\widehat{V^{K}}=\left\{\alpha_{\lambda}: \lambda \in V\right\}$.

By ${ }^{20}$, positive semicharacters lead to deformed convolutions:

Proposition 2.1. Let $\alpha_{0} \in X^{*}$ be a positive semicharacter on the commutative hypergroup $(X, *)$, i.e., $\alpha_{0}(x)>0$ for $x \in X$. Then

$$
\mu \bullet \nu=\alpha_{0}\left(\left(\alpha_{0}^{-1} \mu\right) *\left(\alpha_{0}^{-1} \nu\right)\right) \quad\left(\mu, \nu \in M_{c}(X)\right)
$$

extends uniquely to a bilinear, associative, probability preserving, weakly continuous convolution $\bullet$ on $M_{b}(X)$, and $(X, \bullet)$ becomes a commutative hypergroup with the identity and involution of $(X, *) .(X, \bullet)$ will be called deformation of $(X, *)$ w.r.t. $\alpha_{0}$.

Eq.(2.2) shows that $\mu \mapsto \alpha_{0} \mu$ is an algebra isomorphism between $\left(M_{c}(X), *\right)$ and $\left(M_{c}(X), \bullet\right)$ which for unbounded $\alpha_{0}$ cannot be extended to $M_{b}(X)$; cf. Section 3 .

Many data of $(X, \bullet)$ can be expressed in terms of $\alpha_{0}$ and corresponding data of $(X, *)$. For instance, if $\omega$ is a Haar measure of $(X, *)$, then $\alpha_{0}^{2} \omega$ is a Haar measure of $(X, \bullet)$. Moreover, the mapping $M_{\alpha_{0}}: \alpha \mapsto \alpha / \alpha_{0}$ is a homeomorphism between $(X, *)^{*}$ and $(X, \bullet)^{*}$, and also between $\chi(X, *)$ and $\chi(X, \bullet)$; see ${ }^{20}$ and ${ }^{18}$.

Remark 2.1. Deformation is transitive as follows: Let $(K, \bullet)$ be the deformation of $(K, *)$ w.r.t. $\alpha_{0}$, and let $\beta_{0}$ be a positive semicharacter on $(K, \bullet)$. 
Consider further the deformation $(K, \diamond)$ of $(K, \bullet)$ w.r.t. $\beta_{0}$. The function $\alpha_{0} \beta_{0}$ is a positive semicharacter on $(K, *)$, and $(K, \diamond)$ is the deformation of $(K, *)$ w.r.t. $\alpha_{0} \beta_{0}$. For $\beta_{0}=1 / \alpha_{0}$, one obtains $\diamond=*$.

We next present some examples; for further examples see Section 4.

Example 2.2. Let $(V,\langle.,\rangle$.$) be an n$-dimensional Euclidean vector space, $K$ a compact subgroup of the orthogonal group $O(V)$, and $\left(V^{K}, *\right)$ the associated orbit hypergroup. Fix $\rho \in V$ with $-\rho \in K . \rho$, and consider the function $e_{\rho}(x):=e^{\langle\rho, x\rangle}$ on $V$ and

$$
M_{c}^{\rho, K}(V):=\left\{e_{\rho} \mu: \mu \in M_{c}(V) K \text {-invariant }\right\} .
$$

The multiplicativity of $e_{\rho}$ on $V$ yields that w.r.t. the group convolution on $M_{c}(V)$, we have $e_{\rho} \mu * e_{\rho} \nu=e_{\rho}(\mu * \nu)$. Hence, $M_{c}^{\rho, K}(V)$ is a subalgebra of $M_{b}(V)$, and its norm-closure

$$
M_{b}^{\rho, K}(V):=\overline{M_{c}^{\rho, K}(V)}
$$

a Banach subalgebra. On the other hand, $\alpha_{0}(K . x):=\int_{K} e_{\rho}(k \cdot x) d k(x \in V)$ is a positive semicharacter on $\left(V^{K}, *\right)$; see Example 2.1(2) above as well as Proposition 2.8 of ${ }^{18}$. Proposition 2.8 of 18 also states that for the deformation $\left(V^{K}, \bullet\right)$ of $\left(V^{K}, *\right)$ w.r.t. $\alpha_{0}$, the canonical projection $p$ : $V \rightarrow V^{K}$ induces a probability preserving isometric isomorphism of Banach algebras from $M_{b}^{\rho, K}(V)$ onto $M_{b}\left(V^{K}, \bullet\right)$. In other words, the deformed hypergroup algebra may be regarded as Banach algebra of (not longer $K$ biinvariant) measures on $V$.

Example 2.3. It is well-known that the double coset hypergroup $S L(2, \mathbb{C}) / / S U(2)$ and the orbit hypergroup $\left(\mathbb{R}^{3}\right)^{S O(3)}$ may be identified with $[0, \infty[$, and that the associated hypergroup structures on $[0, \infty[$ are deformations of each other; see ${ }^{1},{ }^{18}$, or ${ }^{20}$.

Here is the higher rank extension of this example:

Example 2.4. Let $G$ be a complex, noncompact, connected semisimple Lie group with finite center and $K$ a maximal compact subgroup. Consider the Cartan decomposition $\mathfrak{g}=\mathfrak{k}+\mathfrak{p}$ of the Lie algebra of $G$, and choose a maximal abelian subalgebra $\mathfrak{a} \subset \mathfrak{p} . K$ acts on $\mathfrak{p}$ via the adjoint representation as a group of orthogonal transformations w.r.t. the Killing form $\langle.,$. as scalar product. Let $W$ be the Weyl group of $K$, which acts on $\mathfrak{a}$ as finite reflection group; here and further on we identify $\mathfrak{a}$ with its dual $\mathfrak{a}^{*}$ via the 
Killing form. Fix some Weyl chamber $\mathfrak{a}_{+} \subset \mathfrak{a}$ and the associated set $\Sigma^{+}$of positive roots. Then the closed chamber $C:=\overline{\mathfrak{a}_{+}}$is a fundamental domain for the action of $W$ on $\mathfrak{a}$, and $C$ can be identified with the orbit hypergroup $\left(\mathfrak{p}^{K}, *\right)$, where a $K$-orbit in $\mathfrak{p}$ corresponds to its representative in $C$.

$C$ can also be identified with the commutative double coset hypergroup $G / / K$ where $x \in C$ corresponds to the double coset $K\left(e^{x}\right) K$. Denote the corresponding convolution by $\bullet$. Using the known formulas for the spherical functions on $G / / K$ and $\mathfrak{p}^{K}$ (see Helgason ${ }^{4}$ ), we proved in 18 that $(G / / K, \bullet)=(C, \bullet)$ is the deformation of the orbit hypergroup $\left(\mathfrak{p}^{K}, *\right)=(C, *)$ w.r.t. the positive semicharacter $\alpha_{-i \rho}$ (in the sense of Example 2.1(2)) with

$$
\rho:=\sum_{\alpha \in \Sigma^{+}} \alpha \in \mathfrak{a}_{+} .
$$

As $-\rho \in K . \rho$, the construction in Example 2.2 shows that $M_{b}(G \| K)$ may be embedded into $M_{b}(\mathfrak{p})$ in an isometric, probability preserving way. Here are the most prominent examples (c.f. Appendix $\mathrm{C}$ of ${ }^{9}$ ).

(1) The $A_{d-1}$-case. $K=S U(d)$ is a maximal compact subgroup of $G=S L(d, \mathbb{C})$. In the Cartan decomposition $\mathfrak{g}=\mathfrak{k}+\mathfrak{p}$ we obtain $\mathfrak{p}$ as the additive group $\mathbf{H}_{d}^{0}$ of all Hermitian $d \times d$-matrices with trace 0 , on which $S U(d)$ acts by conjugation. Moreover, $\mathfrak{a}$ consists of all real diagonal matrices with trace 0 and will be identified with

$$
\left\{x=\left(x_{1}, \ldots, x_{d}\right) \in \mathbb{R}^{d}: \sum_{i} x_{i}=0\right\}
$$

on which the Weyl group acts as the symmetric group $S_{d}$. We thus take

$$
C:=\left\{x=\left(x_{1}, \ldots, x_{d}\right) \in \mathbb{R}^{d}: x_{1} \geq x_{2} \geq \ldots \geq x_{d}, \sum_{i} x_{i}=0\right\} .
$$

Then in particular, $\rho=(d-1, d-3, \ldots,-d+3,-d+1)$.

(2) The $B_{d}$-case. For $d \geq 2$ consider $G=S O(2 d+1, \mathbb{C})$ with maximal compact subgroup $K=S O(2 d+1)$. Here $\mathfrak{a}$ may be identified with $\mathbb{R}^{d}$, and we may choose

$$
C=\left\{x \in \mathbb{R}^{d}: x_{1} \geq x_{2} \geq \cdots \geq x_{d} \geq 0\right\}
$$

with the Weyl group $W \simeq S_{d} \ltimes \mathbb{Z}_{2}^{d}$, and $\rho=(2 d-1,2 d-3, \ldots, 1)$.

(3) The $C_{d}$-case. For $d \geq 3$ let $G=S p(d, \mathbb{C})$ with the maximal compact subgroup $K=S p(2 d+1)$. Here, $\mathfrak{a}=\mathbb{R}^{d}$ with $C$ and $W$ as in the $B_{d}$-case. We have $\rho=(2 d, 2 d-1, \ldots, 2)$. The preceding results on hypergroup deformations imply that the hypergroups 
$S p(d, \mathbb{C}) / / S p(2 d+1)$ and $S O(2 d+1, \mathbb{C}) / / S O(2 d+1)$ are (up to isomorphism) deformations of each other; see also ${ }^{18}$.

(4) The $D_{d}$-case. For $d \geq 4$ let $G=S O(2 d, \mathbb{C})$ with maximal compact subgroup $K=S O(2 d)$. In this case $\mathfrak{a}=\mathbb{R}^{d}$ and we may take

$$
C=\left\{x \in \mathbb{R}^{d}: x_{1} \geq x_{2} \geq \cdots \geq x_{d-1} \geq\left|x_{d}\right|\right\}
$$

with $\rho=(2 d-2,2 d-4, \ldots, 2,0)$.

\section{Deformation of convolution semigroups}

We now always assume that $\alpha_{0}$ is a positive semicharacter on a $\sigma$-compact, second countable commutative hypergroup $(X, *)$ and that $(X, \bullet)$ is the associated deformation. We show how under a natural growth condition, convolution semigroups on $(X, *)$ can be deformed into convolution semigroups on $(X, \bullet)$. To describe this condition, we introduce the spaces

$$
\begin{aligned}
& M_{\alpha_{0}}^{b,+}(X):=\left\{\mu \in M^{b,+}(X): \alpha_{0} \mu \in M^{b,+}(X)\right\}, \\
& M_{\alpha_{0}}^{1}(X):=M^{1}(X) \cap M_{\alpha_{0}}^{b,+}(X)
\end{aligned}
$$

as well as the transformation

$$
R_{\alpha_{0}}: M_{\alpha_{0}}^{b,+}(X) \rightarrow M^{1}(X), \quad \mu \mapsto \frac{1}{\int_{X} \alpha_{0} d \mu} \cdot \alpha_{0} \mu .
$$

Lemma 3.1. Let $\mu, \nu \in M^{b,+}(X)$. Then $\mu * \nu \in M_{\alpha_{0}}^{b,+}(X)$ if and only if $\mu, \nu \in M_{\alpha_{0}}^{b,+}(X)$. Moreover, if one of these conditions holds then

$$
R_{\alpha_{0}}(\mu * \nu)=R_{\alpha_{0}}(\mu) \bullet R_{\alpha_{0}}(\nu) .
$$

Proof. If $\mu, \nu$ have compact support, then the lemma is clear by Eq. (2.2).

In the general case, choose compacta $\left(K_{n}\right)_{n \geq 1}$ in $X$ with $X=\bigcup_{n} K_{n}$ and $K_{n+1} \supset K_{n}$ for $n \in \mathbb{N}$. Put $\mu_{n}:=\left.\mu\right|_{K_{n}}$ and $\nu_{n}:=\left.\nu\right|_{K_{n}}$. As the $\mu_{n} * \nu_{n}$ have compact support, we have

$$
\int_{X} \alpha_{0} d\left(\mu_{n} * \nu_{n}\right)=\int_{X} \int_{X} \alpha_{0}(x * y) d \mu_{n}(x) d \nu_{n}(y)=\int_{X} \alpha_{0} d \mu_{n} \cdot \int_{X} \alpha_{0} d \mu_{n} .
$$

Monotone convergence implies that

$$
\int \alpha_{0} d \mu * \nu=\int_{X} \alpha_{0} d \mu \cdot \int_{X} \alpha_{0} d \mu
$$

where one term is finite if and only if so is the other one. This proves the first part of the lemma. Moreover, if these terms are finite, then the same monotone convergence argument shows that for all $f \in C_{b}(X)$ with $f \geq 0$,

$$
\int f d\left(R_{\alpha_{0}}(\mu * \nu)\right)=\int f d\left(R_{\alpha_{0}}(\mu) \bullet R_{\alpha_{0}}(\nu)\right) .
$$


This implies $R_{\alpha_{0}}(\mu * \nu)=R_{\alpha_{0}}(\mu) \bullet R_{\alpha_{0}}(\nu)$.

Remark 3.1. Notice that the mapping $R_{\alpha_{0}}: M_{\alpha_{0}}^{1}(X) \rightarrow M^{1}(X)$ is not (weakly or vaguely) continuous whenever $\alpha_{0}$ is unbounded. In fact, choose $\left(x_{n}\right)_{n \geq 1} \subset X$ with $\alpha_{0}\left(x_{n}\right) \rightarrow \infty$ and $\alpha_{0}\left(x_{n}\right) \geq 1$. Then the measures $\mu_{n}:=\left(1-\alpha_{0}\left(x_{n}\right)^{-1}\right) \delta_{e}+\alpha_{0}\left(x_{n}\right)^{-1} \delta_{x_{n}}$ tend to $\delta_{e}$ while

$$
R_{\alpha_{0}}\left(\mu_{n}\right)=\frac{1}{2-\alpha_{0}\left(x_{n}\right)^{-1}}\left(\left(1-\alpha_{0}\left(x_{n}\right)^{-1}\right) \delta_{e}+\delta_{x_{n}}\right)
$$

does not tend to $\delta_{e}=R_{\alpha_{0}}\left(\delta_{e}\right)$.

We now investigate convolution semigroups.

Definition 3.1. A family $\left(\mu_{t}\right)_{t \geq 0} \subset M^{1}(X)$ is called a convolution semigroup on $(X, *)$, if $\mu_{0}=\delta_{e}$, if $\mu_{s+t}=\mu_{s} * \mu_{t}$ for $s, t \geq 0$, and if the mapping $\left[0, \infty\left[\rightarrow M^{1}(X), t \mapsto \mu_{t}\right.\right.$ is weakly continuous. It is well-known (see Rentzsch ${ }^{12}$ ) that each convolution semigroup $\left(\mu_{t}\right)_{t \geq 0}$ admits a Lévy measure $\eta \in M^{+}(X \backslash\{e\})$ which is characterized by

$$
\int f d \eta=\lim _{t \rightarrow 0} \frac{1}{t} \int f d \mu_{t} \quad \text { for } \quad f \in C_{c}(X) \text { with } \quad e \notin \operatorname{supp} f .
$$

$\left(\mu_{t}\right)_{t \geq 0}$ is called Gaussian, if $\eta=0$ which is equivalent to saying that for all neighborhoods $U$ of $e \in X, \lim _{t \rightarrow 0} \frac{1}{t} \mu_{t}(X \backslash U)=0$.

We next study under which conditions convolution semigroups on $(X, *)$ can be deformed w.r.t. $\alpha_{0}$. We here need the following condition on $\alpha_{0}$.

Definition 3.2. A positive semicharacter $\alpha_{0}$ on $(X, *)$ is called exponential if there exists a neighborhood $U$ of $e \in X$ and a constant $C>0$ such that for all $x, y \in X$ with $y \in x * U, \alpha_{0}(y) / \alpha_{0}(x) \leq C$.

We conjecture that positive semicharacters are always exponential. Unfortunately we are not able to prove this. However, we present at least some criteria and examples in Section 4 below. The following theorem is motivated by ${ }^{5},{ }^{19}$, where a variant for the group case is studied.

Theorem 3.1. Let $\alpha_{0}$ be an exponential positive semicharacter on $(X, *)$ with $\alpha_{0} \geq 1$. Then the following statements are equivalent for a convolution semigroup $\left(\mu_{t}\right)_{t \geq 0}$ on $(X, *)$ with Lévy measure $\eta$.

(1) $\mu_{t} \in M_{\alpha_{0}}^{1}(X)$ holds for some $t>0$.

(2) $\mu_{t} \in M_{\alpha_{0}}^{1}(X)$ holds for all $t \geq 0$, the mapping $\varphi:[0, \infty[\rightarrow] 0, \infty[$ given by $\varphi(t)=\int \alpha_{0} d \mu_{t}$ is continuous and multiplicative, and $\left(R_{\alpha_{0}}\left(\mu_{t}\right)\right)_{t \geq 0}$ is a convolution semigroup on $(X, \bullet)$. 
(3) For any neighborhood $U$ of $e \in X, \int_{X \backslash U} \alpha_{0} d \eta<\infty$.

If one and hence all of these statements hold, then $\alpha_{0} \eta$ is the Lévy measure of the convolution semigroup $\left(R_{\alpha_{0}}\left(\mu_{t}\right)\right)_{t \geq 0}$ on $(X, \bullet)$.

In particular, Gaussian semigroups on $(X, *)$ always lead to Gaussian semigroups on $(X, \bullet)$.

Proof. (1) $\Longrightarrow(2)$ : Lemma 3.1 implies that $\varphi \geq 1$ is well-defined and multiplicative. To check continuity, we observe that the multiplicativity implies that for $N \in \mathbb{N}$ and $0 \leq s \leq 1 / N, \varphi(s)^{N} \varphi(1-s N)=\varphi(1)$ and hence $\varphi(s) \leq \varphi(1)^{1 / N} \rightarrow 1$ for $N \rightarrow \infty$. Therefore, $\varphi$ is continuous at $t=0$ and hence, as a multiplicative function, on [0, $[$. Using Lemma 3.1 and the fact that the mapping $\left[0, \infty\left[\rightarrow M^{1}(X), t \mapsto R_{\alpha_{0}}\left(\mu_{t}\right)=\varphi(t)^{-1} \alpha_{0} \mu\right.\right.$ is vaguely and hence weakly continuous, we conclude that $\left(R_{\alpha_{0}}\left(\mu_{t}\right)\right)_{t \geq 0}$ is a convolution semigroup on $(X, \bullet)$.

$(2) \Longrightarrow(3)$ : The measure $\rho:=\mathbf{1}_{\left\{\alpha_{0} \geq 2\right\}} \eta \in M^{b,+}(X)$ is the Lévy measure of the Poisson semigroup $\left(\nu_{t}:=e^{-\|\rho\| t} \cdot \exp (t \rho)\right)_{t \geq 0}$, exp denoting the exponential function on the Banach algebra $\left(M_{b}(X), *\right)$. Moreover, it is easy to see that $\eta-\rho$ is the Lévy measure of a further convolution semigroup $\left(\tilde{\nu}_{t}\right)_{t \geq 0}$ with $\mu_{t}=\nu_{t} * \tilde{\nu}_{t}$ for $t \geq 0$. Lemma 3.1 shows that $\nu_{t} \in M_{\alpha_{0}}^{1}(X)$ for $t \geq 0$. As obviously $\rho \leq\left(e^{\|\rho\| t} / t\right) \nu_{t}$ for $t>0$, we obtain $\rho \in M_{\alpha_{0}}^{b,+}(X)$ and thus (3). Furthermore, for $f \in C_{c}(X)$ with $e \notin \operatorname{supp} f$,

$$
\lim _{t \rightarrow 0} \frac{1}{t} \int f d R_{\alpha_{0}}\left(\mu_{t}\right)=\lim _{t \rightarrow 0} \frac{1}{t} \int f \alpha_{0} d \mu_{t}=\int f \alpha_{0} d \eta .
$$

Hence, $\alpha_{0} \eta$ is the Lévy measure of the semigroup $\left(R_{\alpha_{0}}\left(\mu_{t}\right)\right)_{t \geq 0}$ on $(X, \bullet) \square$

The proof of $(3) \Longrightarrow(1)$ is more involved. Recapitulate that for a convolution semigroup $\left(\mu_{t}\right)_{t \geq 0}$ on $(X, *)$, the translation operators $T_{t}(f):=$ $\mu_{t}^{-} * f(t \geq 0)$ form a strongly continuous, positive contraction semigroup on $L^{1}(X, \omega), \omega$ being the Haar measure of $(X, *)$; see $[\mathrm{BH}]$. Let $A$ be its infinitesimal generator with the dense domain $D_{A} \subset L^{1}(X, \omega)$. We have:

Lemma 3.2. Let $\alpha_{0}$ be a positive semicharacter and $\left(\mu_{t}\right)_{t \geq 0}$ a convolution semigroup on $(X, *)$ whose Lévy measure $\eta$ satisfies $\int_{\left\{\alpha_{0} \geq 2\right\}} \alpha_{0} d \eta<\infty$. Then for each neighborhood $U$ of $e \in X$ there exists $f \in C_{c}(X) \cap D_{A}$ with $f \geq 0, f=f^{*} \neq 0$, supp $f \subset U$, and $\int|A f| \alpha_{0} d \omega<\infty$.

Proof. Let $U$ be a compact neighborhood of $e \in X$ with $U^{-}=U$. Then by ${ }^{12}$, there exists $f \in D_{A}$ with $\int_{X} f d \omega=1, f \geq 0, f=f^{*}$, and supp $f \subset U$. 
Let $x \notin U * U$ and $y \in U$. Then $f(x * y)=0$, which means that the translate $f_{x}$ given by $f_{x}(y):=f(x * y)$ satisfies $f_{x}=0$ on $U$, and hence

$$
A f(x)=\lim _{t \rightarrow 0} \frac{1}{t}\left(\mu_{t}^{-} * f_{x}(e)-f_{x}(e)\right)=\int f(x * y) d \eta(y) .
$$

Consequently, by Fubini's theorem,

$$
\begin{aligned}
\int_{X}|A f| \cdot \alpha_{0} d \omega & =\int_{U * U}|A f| \cdot \alpha_{0} d \omega+\int_{X \backslash U * U}|A f| \cdot \alpha_{0} d \omega \\
& \leq \int_{U * U}|A f| \cdot \alpha_{0} d \omega+\int_{X} \int_{X \backslash U * U} f(x * y) \alpha_{0}(x) d \omega(x) d \eta(y) .
\end{aligned}
$$

Now

$$
\begin{aligned}
\int_{X \backslash U * U} f(x * y) \alpha_{0}(x) d \omega(x) & =\int_{X} f(x)\left(\mathbf{1}_{X \backslash U * U} \alpha_{0}\right)(x * \bar{y}) d \omega(x) \\
& \leq \mathbf{1}_{X \backslash U}(\bar{y}) \cdot \int_{X} f(x) \alpha_{0}(x) \alpha_{0}(\bar{y}) d \omega(x) \\
& =\mathbf{1}_{X \backslash U}(y) \alpha_{0}(y) \cdot \int f \alpha_{0} d \omega .
\end{aligned}
$$

As $\int \mathbf{1}_{X \backslash U} \alpha_{0} d \eta<\infty$ by assumption, $\int|A f| \alpha_{0} d \omega<\infty$ as claimed.

$(3) \Longrightarrow(1)$ in the theorem now follows from Lemma 3.2 and the following result.

Lemma 3.3. Let $\alpha_{0}$ be an exponential positive semicharacter with $\alpha_{0} \geq 1$, and $\left(\mu_{t}\right)_{t \geq 0}$ a convolution semigroup on $(X, *)$ with generator $A$. Assume that for each neighborhood $U$ of $e \in X$ there exists $f \in C_{c}(X) \cap D_{A}$ with $f \geq 0, f=f^{*} \neq 0$, supp $f \subset U$ and $\int|A f| \alpha_{0} d \omega<\infty$. Then for all $t \geq 0$, $\int \alpha_{0} d \mu_{t}<\infty$.

Proof. Let $U$ be a neighborhood of $e \in X$ and $C_{1}>0$ a constant with $C_{1} \alpha_{0}(x) \leq \alpha_{0}(z)$ for $x \in X$ and $z \in U * x$. Let $f \in C_{c}(X) \cap D_{A}$ with $f \geq 0$, $f=f^{*} \neq 0$, supp $f \subset U$ and $\int|A f| \alpha_{0} d \omega<\infty$. Then for all $m \in \mathbb{N}$, the functions $\alpha_{m}:=\alpha_{0} \wedge m \in C_{b}(X)$ also satisfy $C_{1} \alpha_{m}(x) \leq \alpha_{m}(z)$ for $x \in X$, $z \in U * x$. Hence, there is a constant $C_{2}>0$ depending on $f$ such that for all $m \in \mathbb{N}$ and $x \in X$,

$$
\alpha_{m}(x) \leq C_{2} \cdot \int \alpha_{m}(x * y) f(y) d \omega(y)=C_{2} \cdot \alpha_{m} * f(x) .
$$

Moreover, as $\alpha_{0} \geq 1$, we have for all $m \in \mathbb{N}$ and $x, y \in X$,

$$
\alpha_{m}(x * y) \leq m \wedge \alpha_{0}(x * y)=m \wedge\left(\alpha_{0}(x) \alpha_{0}(y)\right) \leq \alpha_{m}(x) \alpha_{m}(y) .
$$


Define $h_{m}(t):=\int\left(\mu_{t} * f\right) \cdot \alpha_{m} d \omega=\int \alpha_{m} * f d \mu_{t}$. As $f \in D_{A}$ and $A f \in$ $L^{1}(X, \omega)$ holds, we obtain $\frac{d}{d t} \mu_{t} * f=\mu_{t} * A f$ and hence

$$
h_{m}^{\prime}(t)=\int\left(\mu_{t} * A f\right) \cdot \alpha_{m} d \omega=\iint \alpha_{m}(x * y) A f(y) d \mu_{t}(x) d \omega(y) .
$$

Therefore, by (3.2) and (3.1),

$$
\begin{aligned}
\left|h_{m}^{\prime}(t)\right| & \leq \iint \alpha_{m}(x * y)|A f(y)| d \mu_{t}(x) d \omega(y) \leq \int \alpha_{m} d \mu_{t} \cdot \int \alpha_{m}|A f| d \omega \\
& \leq C_{2} \int \alpha_{m} * f d \mu_{t} \cdot \int \alpha_{m}|A f| d \omega \leq C_{2} \int \alpha_{0}|A f| d \omega \cdot h_{m}(t) .
\end{aligned}
$$

This yields $h_{m}(t) \leq h_{m}(0) e^{t C}$ for $t \geq 0$ and some constant $C \geq 0$ independent of $m$. Hence, again by (3.1),

$$
\int \alpha_{m} d \mu_{t} \leq C_{2} \int \alpha_{m} * f d \mu_{t}=C_{2} h_{m}(t) \leq c_{2} e^{t C} \int \alpha_{0} f d \omega
$$

for all $m \in \mathbb{N}$. This yields the claim $\int \alpha_{0} d \mu_{t}<\infty$ for $t \geq 0$.

Notice that the growth condition on $\alpha_{0}$ was needed above only for the preceding lemma. Theorem 3.1 therefore admits the following variant.

Theorem 3.2. Let $\alpha_{0}$ be a positive semicharacter and $\left(\mu_{t}\right)_{t \geq 0} \subset M^{1}(X)$ a Poisson semigroup on $(X, *)$, which means that $\mu_{t}=e^{-t\|\rho\|} \exp (t \rho)$ for all $t \geq 0$ and some $\rho \in M^{b,+}(X)$. Then $\rho$ is the Lévy measure of $\left(\mu_{t}\right)_{t \geq 0}$, and the statements (1)-(3) of Theorem 3.1 are equivalent.

Proof. It suffices to check (3) $\Longrightarrow(1)$. However, if $R:=\int \alpha_{0} d \rho<\infty$, then for all $n \geq 0, \int \alpha_{0} d \rho^{(n)}=R^{n}$ and hence $\int \alpha_{0} d \mu_{t}<\infty$ for all $t \geq 0$.

Remark 3.2. Let $\alpha_{0}$ be an exponential positive semicharacter and $\left(\mu_{t}\right)_{t \geq 0} \subset M_{\alpha_{0}}^{1}(X)$ a convolution semigroup on $(X, *)$. Then the convolution operators $\left(T_{t}\right)_{t \geq 0}$ on $C_{0}(X)$ with $T_{t} f:=\mu_{t}^{-} * f$ form a Feller semigroup. Its generator $A$ with

$$
A f(x)=\lim _{t \rightarrow 0} \frac{1}{t}\left(\mu_{t}^{-} * f(x)-f(x)\right) \quad(x \in X, f \in D(A))
$$

admits a $\|\cdot\|_{\infty}$-dense domain $D(A)$ in $C_{0}(X)$; see ${ }^{13}$. Now consider the generator $A^{\alpha_{0}}$ of the Feller semigroup on $C_{0}(X)$ which is associated with the renormalized convolution semigroup $\left(R_{\alpha_{0}}\left(\mu_{t}\right)\right)_{t \geq 0}$ on $(X, \bullet)$. Using the notation above, we have

$$
\left(\left(R_{\alpha_{0}} \mu_{t}\right)^{-} \bullet f\right)(x)=\frac{1}{\varphi(t)}\left(\left(\alpha_{0} \mu_{t}\right)^{-} \bullet f\right)(x)=\frac{1}{\varphi(t) \alpha_{0}(x)}\left(\mu_{t} * \alpha_{0} f\right)(x) .
$$


Theorem 3.1(2) shows that $\varphi(t)=e^{c t}$ for some $c \in \mathbb{R}$, and

$$
\lim _{t \rightarrow 0} \frac{1}{t}(1 / \varphi(t)-1)=-c \text {. }
$$

Hence

$$
\begin{aligned}
A^{\alpha_{0}} f(x) & =\lim _{t \rightarrow 0} \frac{1}{t}\left(\frac{1}{\varphi(t) \alpha_{0}(x)}\left(\mu_{t} * \alpha_{0} f\right)(x)-f(x)\right) \\
& =\frac{1}{\alpha_{0}(x)} \lim _{t \rightarrow 0} \frac{1}{t}\left(\frac{1}{\varphi(t)}\left(\mu_{t} * \alpha_{0} f\right)(x)-\left(\alpha_{0} f\right)(x)\right) \\
& =\frac{1}{\alpha_{0}(x)} A\left(\alpha_{0} f\right)(x)+\frac{1}{\alpha_{0}(x)} \lim _{t \rightarrow 0}\left(\frac{1}{t}(1 / h(t)-1)\left(\mu_{t} * \alpha_{0} f\right)(x)\right) \\
& =\frac{1}{\alpha_{0}(x)} A\left(\alpha_{0} f\right)(x)-c f(x) .
\end{aligned}
$$

Therefore

$$
A^{\alpha_{0}}=M_{1 / \alpha_{0}} \circ A \circ M_{\alpha_{0}}-c
$$

at least on $D\left(A^{\alpha_{0}}\right) \cap C_{c}(X)$, where $M_{g}$ denotes the multiplication operator with $g \in C(K)$. The same holds for other function spaces like $L^{p}(X, \omega)$.

\section{Exponential positive semicharacters}

It seems reasonable to conjecture that positive semicharacters are always exponential. Unfortunately we are not able to prove this. Here are, at least, some criteria and several examples:

\section{Lemma 4.1.}

(1) If $(X, *)$ is discrete, then $\alpha_{0}$ is always exponential.

(2) Let $\alpha_{0}, \alpha_{1}$ be exponential positive semicharacters on $(X, *)$, and let $(X, \bullet)$ be the deformation of $(X, *)$ w.r.t. $\alpha_{0}$. Then $\alpha_{1} / \alpha_{0}$ is an exponential positive semicharacter on $(X, \bullet)$.

Proof. Part (1) is clear by taking $U=\{e\}$. For the proof of (2) choose neighborhoods $U_{0}, U_{1}$ of $e$ and constants $C_{0}, C_{1}$ associated with $\alpha_{0}, \alpha_{1}$ respectively. For $U:=U_{0} \cap U_{1} \cap U_{0}^{-} \cap U_{1}^{-}$and $C:=C_{0} C_{1}$, we obtain that for $x, y \in X$ with $y \in x * U$, we have $x \in y * U$ and thus $\alpha_{0}(x) \alpha_{1}(y) /\left(\alpha_{0}(y) \alpha_{1}(x)\right) \leq C$ as claimed.

Example 4.1. In ${ }^{23}$, Zeuner presented quite general, but technical conditions on a function $A \in C\left(\left[0, \infty[) \cap C^{1}(] 0, \infty[)\right.\right.$ with $A(x)>0$ for $x \geq 0$ which ensures that there exists a unique commutative hypergroup $([0, \infty[, *)$ 
whose semicharacters are precisely the eigenfunctions of the Sturm-Liouville operator

$$
L_{A} f:=-f^{\prime \prime}-\left(A^{\prime} / A\right) f^{\prime}
$$

with initial conditions $f(0)=1$ and $f^{\prime}(0)=0$; see also Section 3.5 of ${ }^{1}$. This hypergroup is called the Sturm-Liouville hypergroup associated with $A$. Moreover, to the knowledge of the authors, all known hypergroup structures on $\left[0, \infty\right.$ [ appear in this way (up to isomorphism); see also ${ }^{1}$ for details. We here mention that Zeuner's approach in particular includes all Chebli-Trimeche hypergroups and thus all double coset hypergroups associated with noncompact symmetric spaces of rank one.

We claim that all positive semicharacters on a Sturm-Liouville hypergroup on $[0, \infty[$ with $A$ satisfying Zeuner's conditions are exponential. To prove this, recall from Section 3.5 in ${ }^{1}$ that Zeuner's conditions imply that

$$
\rho:=\frac{1}{2} \lim _{x \rightarrow \infty} A^{\prime}(x) / A(x) \geq 0
$$

exists, and that the positive semicharacters are precisely the unique solutions $\varphi_{i \lambda}$ of

$$
L_{A} \varphi_{i \lambda}=\left(\rho^{2}-\lambda^{2}\right) \varphi_{i \lambda}, \quad \varphi_{i \lambda}(0)=1, \varphi_{i \lambda}^{\prime}(0)=0
$$

with $\lambda \geq 0$. Moreover, the renormalization $([0, \infty[, \bullet)$ of $([0, \infty[, *)$ w.r.t. $\varphi_{i \lambda}$ is again a Sturm-Liouville hypergroup associated with the renormalized function $A_{\lambda}:=\varphi_{i \lambda}^{2} \cdot A$ where $A_{\lambda}$ again satisfies Zeuner's conditions; see Section 3.5.51 of ${ }^{1}$. Applying (4.1) to $A$ as well as to $A_{\lambda}$, we see that $\lim _{x \rightarrow \infty} \varphi_{i \lambda}^{\prime}(x) / \varphi_{i \lambda}(x)$ exists. As $\operatorname{supp}\left(\delta_{x} * \delta_{y}\right) \subset[|x-y|, x+y]$ for $x, y \geq 0$, it follows from the mean-value theorem that $\varphi_{i \lambda}$ is exponential.

Example 4.2. Let $V$ be a finite-dimensional Euclidean vector space, $K \subset$ $O(V)$ a compact subgroup, and $V^{K}$ the associated orbit hypergroup as in Example 2.1(2). Then, for each $\rho \in V$, the positive semicharacter $\alpha_{i \rho}$ with $\alpha_{i \rho}(K . x)=\int_{K} e^{-\langle\rho, k . x\rangle} d k(x \in V)$ is exponential. In fact, we may take $U:=\left\{K . x: x \in V,\|x\|_{2} \leq 1\right\} \subset V^{K}$ as a neighborhood of the identity. For orbits $K . x, K . y \in V^{K}$ with $K . x \in U * K . y$ we then have representatives $x, y \in V$ with $\|x-y\|_{2} \leq 1$ which implies that $e^{-\langle\rho, k . x\rangle} \leq e^{-\langle\rho, k . y\rangle} e^{\|\rho\|_{2}}$ for $k \in K$ and thus $\alpha_{i \rho}(K . x) \leq \alpha_{i \rho}(K . y) e^{\|\rho\|_{2}}$ as claimed.

Example 4.3. Let $G$ be a (not necessarily complex) noncompact, connected semisimple Lie group with finite center and $K$ a maximal compact subgroup. Let $G=N A K$ and $\mathfrak{g}=\mathfrak{n}+\mathfrak{a}+\mathfrak{k}$ be the corresponding Iwasawa decompositions. For $g \in G$ let $A(g) \in \mathfrak{a}$ be the unique element with 
$g \in N \exp (A(g)) K$. Let $\Sigma^{+}$be the set of positive roots (for the order corresponding to $\mathfrak{n}$ ), and $\rho=\frac{1}{2} \sum_{\alpha \in \Sigma^{+}} m_{\alpha} \alpha$ the half sum of positive roots with $m_{\alpha}$ as multiplicity of $\alpha$. Then, by a formula of Harish-Chandra (see Theorem IV.4.3 of ${ }^{4}$ ), the spherical functions on $G$, i.e., the multiplicative functions on $G / / K$, are given by

$$
\varphi_{\lambda}(g)=\int_{K} e^{\langle i \lambda+\rho, A(k g)\rangle} d k \quad(g \in G),
$$

where $\lambda$ runs through $\mathfrak{a}_{\mathbb{C}}$, the complexification of $\mathfrak{a}$. Clearly, the $\varphi_{\lambda}$ for $\lambda \in i \cdot \mathfrak{a}$ are positive multiplicative functions. These functions are also exponential. To prove this, we conclude from Lemma IV.4.4 of ${ }^{4}$ that

$$
\varphi_{\lambda}\left(g^{-1} h\right)=\int_{K} e^{\langle-i \lambda+\rho, A(k g)\rangle} e^{\langle i \lambda+\rho, A(k h)\rangle} d k \quad(g, h \in G) .
$$

Hence, for each compact neighborhood $U$ of $e$ there is a constant $C>0$ such that $\varphi_{\lambda}\left(g^{-1} h\right) \leq C \varphi_{\lambda}(h)$ for all $g \in U, h \in G$ and $\lambda \in i \cdot \mathfrak{a}$.

Example 4.4. Let $R$ be a (reduced, not necessaryly crystallographic) root system in $\mathbb{R}^{n}$ with the standard inner product $\langle.$, . $\rangle$, i.e. $R \subset \mathbb{R}^{n} \backslash\{0\}$ is finite with $R \cap \mathbb{R} \alpha=\{ \pm \alpha\}$ and $\sigma_{\alpha}(R)=R$ for all $\alpha \in R$, where $\sigma_{\alpha}$ is the reflection in the hyperplane perpendicular to $\alpha$. Assume also without loss of generality for our considerations that $\langle\alpha, \alpha\rangle=2$ for all $\alpha \in R$. Let $W$ be the finite reflection group generated by the $\sigma_{\alpha}$ and let $k: R \rightarrow[0, \infty[$ be a fixed multiplicity function on $R$, i.e. a function which is constant on the orbits under the action of $W$. The (so-called rational) Dunkl operators attached to $G$ and $k$ are defined by

$$
T_{\xi}(k) f(x)=\partial_{\xi} f(x)+\sum_{\alpha \in R_{+}} k(\alpha)\langle\alpha, \xi\rangle \frac{f(x)-f\left(\sigma_{\alpha} x\right)}{\langle\alpha, x\rangle}, \quad x, \xi \in \mathbb{R}^{n} .
$$

Here $\partial_{\xi}$ denotes the derivative in direction $\xi$ and $R_{+}$is some fixed positive subsystem of $R$. The definition is independent of the special choice of $R_{+}$, due to the $G$-invariance of $k$. As first shown in ${ }^{2}$, the $T_{\xi}(k), \xi \in \mathbb{R}^{n}$ generate a commutative algebra of differential-reflection operators. This is the foundation for rich analytic structures related with them. In particular, there exists a counterpart of the exponential function, the Dunkl kernel, and an analogue of the Euclidean Fourier transform with respect to this kernel. The Dunkl kernel $E_{k}$ is holomorphic on $\mathbb{C}^{n} \times \mathbb{C}^{n}$ and symmetric in its arguments. Similar to spherical functions on symmetric spaces, the function $E_{k}(., y)$ with fixed $y \in \mathbb{C}^{n}$ may be characterized as unique analytic solution of the joint eigenvalue problem

$$
T_{\xi}(k) f=\langle\xi, y\rangle f \quad \text { for all } \xi \in \mathbb{C}^{n}, f(0)=1 ;
$$


c.f. ${ }^{11}$. Apart from the trivial case $k=0$ with $E_{k}(x, y)=e^{\langle x, y\rangle}, E_{k}$ is explicitly known in a few cases only like $n=1$; see ${ }^{17}$ for a survey. The $G$-invariant counterpart of $E_{k}$ is the generalized Bessel function

$$
J_{k}(x, y)=\frac{1}{|G|} \sum_{g \in G} E_{k}(g x, y)
$$

which is $G$-invariant in $x, y$ and naturally considered on the closed positive Weyl chamber $C$ associated with $R_{+}$. For $n=1, J_{k}$ is a usual Bessel function. Moreover, in the cristallographic case and for certains half-integer multiplicities, the $J_{k}$ are the multiplicative functions of certain Euclidean orbit hypergroups as in Example 2.1. Here, and for $n=1$, the $J_{k}(x, y)$ $\left(y \in \mathbb{C}^{n}\right)$ therefore form the multiplicative functions of some commutative hypergroup on $C$. It is conjectured that there exist such commutative hypergroups on $C$ for all root systems and multiplicities $k \geq 0$. Only part of this conjecture has been verified up to now in ${ }^{16}$.

Now fix a root system $R$ and $k \geq 0$ such that the $J_{k}(., y)\left(y \in \mathbb{C}^{n}\right)$ are the multiplicative functions of a commutative hypergroup $(C, *)$. To find positive semicharacters, we employ the following psoitive integral representation for $E_{k}$ (and thus $J_{k}$ ): For given $R, k \geq 0$, and $x \in \mathbb{R}^{n}$ there exists a unique pribability measure $\mu_{x}$ on $\mathbb{R}^{n}$ such that

$$
J_{k}(x, y)=\int e^{\langle z, y\rangle} d \mu_{x}(z) \quad \text { for } \quad y \in \mathbb{C}^{n} .
$$

Moreover, supp $\mu_{x} \subset\left\{z \in \mathbb{R}^{n}:\|z\|_{2} \leq\|x\|_{2}\right\}$. Thus, for each $y \in \mathbb{R}^{n}$, $J_{k}(., y)$ is a positive semicharacter on $(C, *)$. We claim that these semicharacters are exponential.

To show this, let $U:=\left\{z \in C:\|z\|_{2} \leq 1\right\}$ and $x_{1}, x_{2} \in C$ with $x_{1} \in$ $U * x_{2}$. We conclude from Theorem 4.1 of ${ }^{16}$ that then $x_{1} \in C \cap \bigcap_{w \in W}\{z \in$ $\left.\mathbb{R}^{n}:\left|z-w \cdot x_{2}\right| \leq 1\right\}$ holds. As $\|z-w\| \leq\|z-w \cdot x\|$ for all $x, z \in C$ and $w \in W$ by Ch. 3 of ${ }^{3}$, we even have $\left\|x_{1}-x_{2}\right\| \leq 1$. In the same way as in Example 4.3 we now obtain from Eq. (4.4) that $J_{k}\left(x_{1}, y\right) \leq e^{\|y\|} J_{k}\left(x_{2}, y\right)$ which proves that $J_{k}(., y)$ is exponential for each $y \in \mathbb{R}^{n}$.

\section{References}

1. W. Bloom, H. Heyer, Harmonic Analysis of Probability Measures on Hypergroups. De Gruyter-Verlag, Berlin, 1994.

2. C.F. Dunkl, Differential-difference operators associated to reflection groups, Trans. Amer. Math. Soc. 311 (1989), 167-183.

3. L.C. Grove, C.T. Benson, Finite Reflection Groups. Springer-Verlag, 2nd ed., 1985. 
4. S. Helgason, Groups and Geometric Analysis. American Mathematical Society, 2000.

5. A. Hulanicki, A class of convolution semi-groups of measures on a Lie group. In: Probab. Theory on Vector Spaces II, Proceedings. Lecture Notes in Mathematics Vol. 828, Springer 1980, pp. 82-101.

6. R.I. Jewett, Spaces with an abstract convolution of measures. Adv. Math. 18 (1975), 1-101.

7. A. Klyachko, Stable bundles, representation theory, and Hermitian operators, Selecta Math. (new Series) 4 (1998), 419-445.

8. A. Klyachko, Random walks on symmetric spaces and inequalities for matrix spectra, Linear Algebra Appl. 319 (2000), 37-59.

9. A.W. Knapp, Lie Groups beyond an Introduction. Birkhäuser, Boston, 1996.

10. A. Knutson, T. Tao, The honeycomb model of $G L_{n}(\mathbb{C})$ tensor product $I$. Proof of the saturation conjecture, J. Amer. Math. Soc. 12 (1999), 10551090.

11. E.M. Opdam, Dunkl operators, Bessel functions and the discriminant of a finite Coxeter group, Compos. Math. 85 (1993), 333-373.

12. C. Rentzsch, A Lévy-Khintchine type representation of convolution semigroups on commutative hypergroups. Probab. Math. Stat. 18 (1998), 185198.

13. C. Rentzsch, M. Voit, Lévy processes on commutative hypergroups. Contemp. Math. 261 (2000), 83-105.

14. L.C.G. Rogers, D. Williams, Diffusions, Markov Processes, and Martingales, Vol. II. Wiley: Chichester - New York, 1987.

15. M. Rösler, Positivity of Dunkl's intertwining operator. Duke Math. J. 98 (1999), 445-463.

16. M. Rösler, A positive radial product formula for the Dunkl kernel. Trans. Amer. Math. Soc. 355 (2003), 2413-2438.

17. M. Rösler, Dunkl operators: Theory and Applications. Lecture Notes for the SIAM Euro Summer School: Orthogonal Polynomials and Special Functions. (Leuven, 2002). In: Springer Lecture Notes in Mathematics, Vol. 1817, 2003, pp. $93-136$.

18. M. Rösler, M. Voit, $S U(d)$-biinvariant random walks on $S L(d, \mathbb{C})$ and their Euclidean counterparts, preprint 2003.

19. E. Siebert, Continuous convolution semigroups integrating a submultiplicative function, Man. Math. 37 (1982), 383-391.

20. M. Voit, Positive characters on commutative hypergroups and some applications, Math. Z. 198 (1988), 405-421.

21. M. Voit, A Girsanov-type formula for Lévy processes on commutative hypergroups. In: H. Heyer et al. (eds.), Infinite Dimensional Harmonic Analysis, Proc. Conf., Gräbner 2000, pp. 346-359.

22. H. Zeuner, The central limit theorem for Chébli-Trimeche hypergroups. J. Theor. Probab. 2 (1989), 51-63.

23. H. Zeuner, Moment functions and laws of large numbers on hypergroups. Math. Z. 211 (1992), 369-407. 